\title{
Atom diffusion in a simple cubic lattice: Real-space renormalization group and Monte Carlo calculations
}

\author{
N. Tarasenko, ${ }^{1}$ L. Jastrabik, ${ }^{1}$ T. Müller, ${ }^{2}$ and A. Tarasenko ${ }^{1,3, *}$ \\ ${ }^{1}$ Institute of Physics, Academy of Sciences of the Czech Republic, Na Slovance 2, 182 21, Prague 8, Czech Republic \\ ${ }^{2}$ Central Institute for Applied Mathematics, Research Centre Jülich, D-52425, Jülich, Germany \\ ${ }^{3}$ Institute of Physics, National Academy of Sciences of Ukraine, Prospect Nauki 46, UA-03039, Kyjiv 39, Ukraine
}

(Received 27 November 2005; revised manuscript received 15 March 2006; published 19 June 2006)

\begin{abstract}
A three-dimensional simple cubic lattice gas is investigated by using the real-space renormalization group (RSRG) approach with blocks of different size and symmetries. We have calculated absorption isotherms and density dependencies of the pair correlation function, isothermal susceptibility, chemical and jump diffusion coefficients for different temperatures. All these quantities have also been obtained by Monte Carlo (MC) simulations. We have also obtained the density dependencies for the tracer diffusion coefficient by MC simulations. Despite the fact that both methods constitute very different approaches, the correspondence of numerical data and theoretical results is rather good. We conclude that the RSRG method can be applied to the three-dimensional lattice gas system discussed here to characterize its thermodynamic and kinetic properties.
\end{abstract}

DOI: 10.1103/PhysRevB.73.245419

PACS number(s): 64.60.Ak, 66.10.Cb, 64.60.Cn, 66.30.Dn

\section{INTRODUCTION}

The migration of particles (atoms, molecules, ions) plays an essential role in many physical and chemical processes such as melting, roughening, crystal and film growth, and ionic transport in solids to name a few. The understanding of mechanisms of particle migration through solids is of fundamental importance in many branches of material sciences. Details of the migration processes is the primary aim of the theory in this area. Appropriate models should reflect the elementary microscopic migration acts of particles, which depend on the structure and mutual particle-particle interaction. To include all these aspects it is most convenient to employ the lattice gas model. In this model particles perform stochastic jumps among sites of a discrete lattice. These lattice sites represent the interstitial sites preferred by the particles in the host material. During the elementary act of migration, affected by thermal activation, a particle has to surmount the barrier separating the sites. The effective barrier height depends on the specific atomic environment and, as a consequence of the particle-particle interaction, also on the number and configuration of the neighbor particles.

The effects of lateral interactions on the particle diffusion have been intensively investigated using the most refined analytical and MC techniques. ${ }^{1-9}$ It has been found that particle interaction can strongly influence diffusion, especially at low temperatures and in the vicinity of phase transitions.

In general, the determination of the diffusion coefficient requires the solution of a kinetic equation for a many-particle system. However, under simplifying assumptions such as slowly varying particle density inhomogeneities and neglecting memory effects, the problem can be reduced to the calculation of purely thermodynamic quantities: Free energy and its derivatives over chemical potential and interaction parameters. ${ }^{10-12}$ This approach has been used widely for the theoretical treatment of particle diffusion. The task of computing thermodynamic quantities is substantially simpler and computationally less expensive by orders of magnitude than direct MC simulations of kinetic phenomena which suffer from the statistical uncertainty of the results. It turns out that the approximations involved when connecting diffusion coefficients with thermodynamical quantities are rather reliable in the case of two-dimensional lattice gas systems. Results obtained by different analytical methods (quasi-chemical approximation, cluster variation approach, and the RSRG method) show quite good agreement with the numerical data (kinetic MC simulations). Yet there is no general upper bound to the errors established for these approximations just as there is no guarantee for the $\mathrm{MC}$ results to converge to the required statistical accuracy for the general case with limited computer resources. Compared to purely numerical schemes, the theoretical methods of statistical mechanics reveal correlations between the kinetic coefficients and some thermodynamical quantities thereby providing the basis for additional insight.

The diffusion in a simple cubic lattice was investigated for the cases of strong attractive ${ }^{13}$ and strong repulsive $e^{14,15}$ lateral particle interactions where the system of particles is in the inhomogeneous or ordered state, respectively.

In the present work we have investigated particle diffusion by means of the RSRG approach and MC simulations considering temperatures mainly above the critical point. For calculating the thermodynamical quantities which enter the approximate expression for the diffusion coefficient a variety of analytical methods can be used. Among them the RSRG approach is particularly simple so that a rather modest effort suffices to compute data of good accuracy. This applies in particular to the two-dimensional lattice gas systems.

We have analyzed a number of RSRG transformations with different blocks of lattice sites. We have calculated RSRG transformation matrices and determined their critical properties. Calculations of the diffusion coefficients is a nice possibility to check the applicability and correctness of these transformations as one should obtain the first derivatives of the free energy over chemical potential and interaction parameter and the second derivative over the chemical potential. We present density dependencies of the jump and chemical diffusion coefficients along with the isothermal 
susceptibility. Comparing all these quantities with the corresponding MC data reveals good overall agreement for the results of these entirely different methods.

\section{DIFFUSION OF PARTICLES IN A SIMPLE CUBIC LATTICE}

We consider an array of absorption sites arranged in a simple cubic lattice. The potential energy landscape is assumed to consist of pits of depth $\varepsilon$ located at the sites which the particles are solely allowed to occupy. Due to the thermal activation, particles jump from time to time to the empty nearest neighbor $(n n)$ sites. The state of the system of absorbed particles is described by a set of time-dependent occupation numbers, $\left\{n_{i}\right\}$, as follows:

$$
n_{i}= \begin{cases}1, & \text { if site } i \text { is occupied, } \\ 0, & \text { if site } i \text { is empty. }\end{cases}
$$

In thermodynamic equilibrium the description of the system behavior is reduced to the calculation of the grand partition function

$$
Q=\sum_{\left\{n_{i}\right\}} \exp \left(\mu N_{a}-H_{a}\right)
$$

or its corresponding potential, $F=N^{-1} \ln Q$, which we will call free energy. Here $\mu, N_{a}=\sum_{i} n_{i}$ and $H_{a}$ denote the chemical potential, number of particles and Hamiltonian of the system, respectively; the summation is carried out over all particle configurations (here and henceforth we use the system of units with $\left.k_{B} T=1\right)$. The Hamiltonian can be written in the form

$$
H_{a}=-\varepsilon N_{a}+\varphi \sum_{\langle n n\rangle} n_{i} n_{j},
$$

where $\varphi$ is the pair interaction energy of $n n$ particles; symbol $\langle n n\rangle$ denotes summation over all lattice bonds just once.

Several diffusion coefficients had been defined to describe the particle migration. Conceptually the simplest diffusion coefficient is a single particle or tracer diffusion coefficient, $D_{t}$, which is related to the asymptotic behavior of the mean square displacements of the tagged particles.

$$
D_{t}=\lim _{t \rightarrow \infty} \frac{1}{2 d N_{a}} \sum_{i=1}^{N_{a}}\left\langle\left[\mathbf{r}_{i}(t)-\mathbf{r}_{i}(0)\right]^{2}\right\rangle,
$$

where $d=3$ is the system dimension; $\mathbf{r}_{i}(t)$ is the position of the $i$ th particle at time $t$.

The jump diffusion coefficient $D_{j}$ is a many particle kinetic coefficient describing the asymptotic behavior of the mean square displacement of the system center of mass.

$$
D_{j}=\lim _{t \rightarrow \infty} \frac{1}{2 d N_{a} t}\left\langle\left\{\sum_{i=1}^{N_{a}}\left[\mathbf{r}_{i}(t)-\mathbf{r}_{i}(0)\right]\right\}^{2}\right\rangle,
$$

The definitions for $D_{t}$ and $D_{j}$ are well-suited for MC simulations as they are expressed in terms of the directly accessible quantities.

The chemical diffusion coefficient is determined by Fick's first law, which constitutes a linear relationship between the

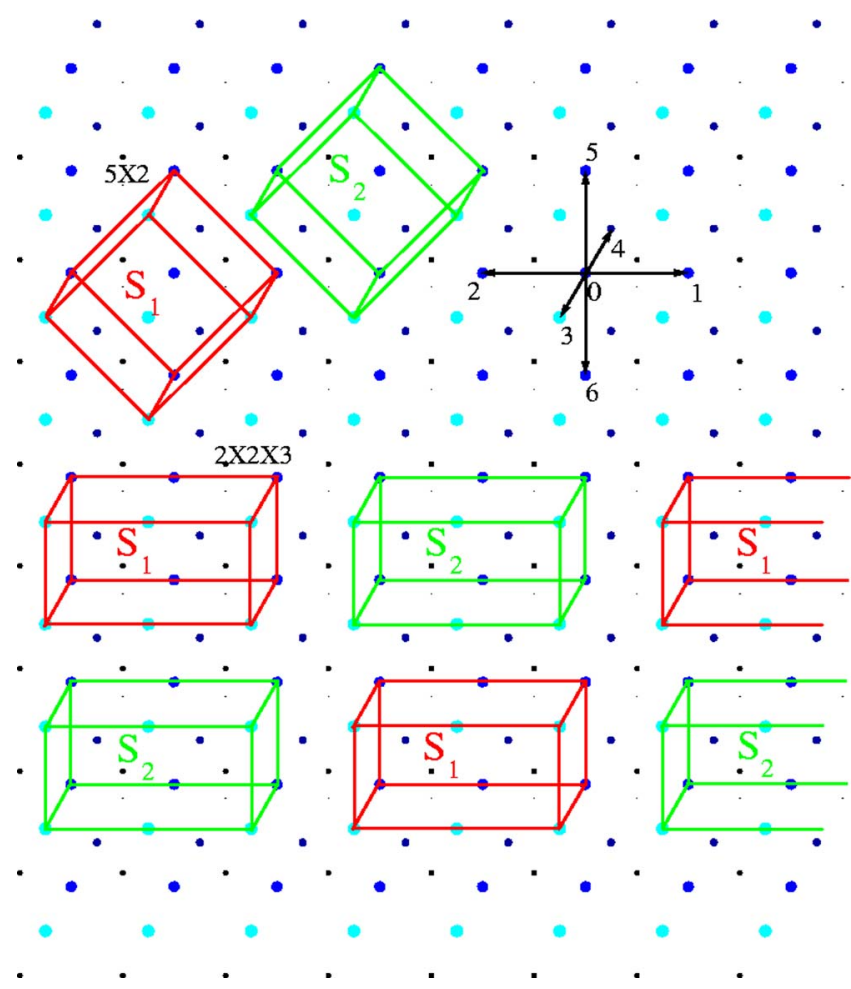

FIG. 1. (Color online) Simple cubic lattice with two different kinds of the RSRG blocks. Arrows show six possible jumps of a particle out of the zeroth site.

flux of particles $\mathbf{J}(\mathbf{r}, t)$ and the gradient of the particle density $\theta(\mathbf{r}, t)$ in the following way:

$$
\mathbf{J}(\mathbf{r}, t)=-D_{c} \boldsymbol{\nabla} \theta(\mathbf{r}, t) .
$$

To describe particle diffusion one must resolve the diffusion process into its elementary components. Particle transport may be described in terms of jumps of particles in a regular or random energy landscape. Particles perform random walks jumping over potential energy pits of a simple cubic lattice. A particle can jump to any of its unoccupied $n n$ sites (labeled 1, .,6 in Fig. 1) thereby surmounting the potential barrier $E_{i f}$ between the initial (ith) and final $(f$ th) sites. For the Langmuir lattice gas $\varphi=0$ and potential barrier is a constant $\varepsilon$ throughout the entire lattice. For the interacting lattice gas the activation energy depends on the number of adjacent particles. We assume that the interactions affect the minima of the potential landscape and neglect the influence of these interactions on an activated particle at the saddle point of the potential barrier. Then, the jump frequency from the initial site to the final site has the following form:

$$
\nu_{i f}=\nu \exp \left\{-\varepsilon+\varphi \sum_{k=1}^{6} n_{k}\right\},
$$

where summation runs over all $n n$ s of the initial site. The jump frequency depends on the energy of the initial state only and is the same for jumps to all $n n$ sites. The model should be appropriate for a short range lateral interaction 
between the particles. It is certainly important to note, that Eq. (7) represents a mathematical recipe or transition algorithm describing how particles move in the lattice. Different algorithms are conceivable and have been used in the diffusion theory and MC simulations, some of them are described in Ref. 16.

In order to obtain a suitable expression for $D_{c}$ we have used the local equilibrium approximation and neglected the memory effects (interested readers can find details of the derivation in Refs. 5, 10, and 12). In this approximation the chemical diffusion coefficient is given by

$$
D_{c}=D_{0} \exp (\mu) P_{00} / \chi_{T},
$$

where $D_{0}=3 \nu \exp (-\varepsilon) a^{2} / 2$ is the diffusion coefficient of the noninteracting particles, $P_{00}$ denotes the probability to find a pair of empty $n n$ sites, $\chi_{T}$ is the isothermal susceptibility or mean-square particle density fluctuation. The jump and chemical diffusion coefficients are simply related via the Kubo-Green equation

$$
D_{c}=\theta D_{j} / \chi_{T} .
$$

where $\theta=\left\langle n_{0}\right\rangle$ denotes the mean particle density. For the Langmuir lattice gas the relations between the diffusion coefficients are following: $D_{t}=D_{j}=(1-\theta) D_{0}$ and $D_{c}=D_{0}$.

Equation (8) is rather simple. However, it gives a good description of the particle diffusion in the case of twodimensional honeycomb, square, and triangular lattices. ${ }^{5-7}$ Its specific property is the presence of the pure thermodynamic quantities only on the right-hand side. The kinetic quantity-chemical diffusion coefficient-is expressed via the first and second derivatives of the free energy

$$
\begin{gathered}
\theta=\frac{\partial F}{\partial \mu}, \quad \chi_{T}=\frac{\partial^{2} F}{\partial \mu^{2}}, \quad\left\langle n_{0} n_{1}\right\rangle=-\frac{2}{z} \cdot \frac{\partial F}{\partial \varphi}, \\
P_{00} \equiv\left\langle\left(1-n_{0}\right)\left(1-n_{1}\right)\right\rangle=1-2 \theta+\left\langle n_{0} n_{1}\right\rangle,
\end{gathered}
$$

where $z=6$ is the coordination number of the lattice.

Thus, the calculation of $D_{c}$ is reduced to the determination of the lattice gas free energy, $F$. To determine the free energy of the system one needs to use some approximations. Even for the simplest lattice gas model, the problem remains too complex to be solved exactly. In the next section we outline briefly the RSRG method used for this purpose.

\section{RSRG TRANSFORMATIONS IN SIMPLE CUBIC LATTICE}

It is well known that the lattice-gas model with the $n n$ interaction only is equivalent to the spin model with an external magnetic field. Empty sites are equivalent to $s=-1$, and filled sites to $s=1$. Using the obvious linear relation between the spin variables and occupation numbers $n_{i}=(1$ $\left.+s_{i}\right) / 2$, one can easily obtain the equivalent reduced Hamiltonian of the spin model

$$
H(s)=h \sum_{i} s_{i}+k \sum_{\langle n n\rangle} s_{i} s_{j}+N c .
$$

Here

$$
\begin{gathered}
c=(\mu+\varepsilon-3 \varphi / 2) / 2, \\
h=(\mu+\varepsilon-3 \varphi) / 2, \\
k=-\varphi / 4 .
\end{gathered}
$$

The case $\varphi<0$ corresponds to the ferromagnetic (F) spinspin interaction and $\varphi>0$ describes antiferromagnetic (AF) spin model. Strong lateral interaction causes phase transitions. F interaction aligns all spins up or down. For AF ordering one must imagine the origin lattice decomposed into two equivalent sublattices such that all $n n$ s of a given site lie on the opposite sublattice. Then the AF interaction aligns spins on different sublattices in the opposite direction. In lattice gas systems the $\mathrm{F}$ interaction corresponds to attraction and a phase transition of the first order means a jump between a low density $\left(\theta_{c}<1 / 2\right)$ disordered phase (lattice gas) and a high density $\left(1-\theta_{c}\right)$ phase (lattice liquid) at some critical value of the chemical potential $\mu_{c}$, when temperature is below critical. The AF interaction corresponds to the repulsive lateral interaction. The phase transition is of the second order. It causes particles, which occupy lattice sites randomly, to collect on one sublattice leaving the other sublattice empty. The average density does not change during the AF phase transition.

The accurate critical value of the interaction parameter in the absence of an external magnetic field was obtained by means of the MC method and finite size scaling ${ }^{17}$

$$
k_{c} \approx \pm 0.22165455 \text {. }
$$

Although the lattice-gas and spin models are fully equivalent, we prefer to use the spin representation here because of its symmetry with respect to the magnetic field. However, we will refer to lattice-gas terms where this seems to be more appropriate.

In the RSRG method, developed by Niemeyer and van Leeuwen ${ }^{18}$ and Nauenberg and Nienhuis, ${ }^{19-22}$ the whole lattice is divided into blocks (or cells) of $L$ sites. A block spin $S_{i}$ is assigned to each block. The RSRG transformation of the spin system allows the reduction of the number of independent variables, i.e., the transition from the set of $N$ site spins $\left\{s_{i}\right\}$ to $N / L$ block spins $\left\{S_{i}\right\}$. The transformation can be written as follows:

$$
H(S)+g=\ln \sum_{\{s\}} P(S, s) \exp [H(s)],
$$

where $H(S)$ is the renormalized Hamiltonian of the block spin system, $g$ is the "empty set" term which is of great importance in the RSRG method and $P(S, s)$ is the weighting factor. We note that two values of the block spin $S_{i}= \pm 1$ corresponds to $2^{L}$ site spin configurations (since $L$ spins are combined to form a block). Using the weighting factor one can distribute the configurations into domains, corresponding definite values of the block spin. For blocks with odd number 
of spins the value of a block spin $S_{i}$ is usually determined by the so-called majority rule ${ }^{21}$

$$
S_{i}=\operatorname{sgn}\left(\sum_{j=1}^{L} s_{j}\right) \quad \text { where } \operatorname{sgn}(x)= \begin{cases}+1, & \text { if } x>0 \\ -1, & \text { if } x<0 .\end{cases}
$$

For even $L$ assigning a definite value of the block spin to configurations having zero sum of site spins is rather arbitrary. The purpose of any RSRG transformation is to present the result of the summation of Eq. (14) in the same form as the original Hamiltonian Eq. (11) (plus possibly terms which do not significantly affect the critical behavior of the system. In order to carry out the summation in Eq. (14) some approximation must be used. In the framework of the RSRG approach, one usually employs periodic boundary conditions. It is assumed that the whole lattice is given by the periodic continuation of a small cluster of blocks. In the present work we consider the smallest possible cluster of two blocks. Then renormalized $(H, K)$ and original $(h, k)$ values of the interaction parameters of the Hamiltonian are related by

$$
H\left(S_{1}+S_{2}\right)+6 K S_{1} S_{2}+2 L g=\Psi\left(S_{1}, S_{2}\right) .
$$

Here $\Psi\left(S_{1}, S_{2}\right)$ is the function on the right-hand side of Eq. (14) where the summation runs over all configurations $\left\{s_{i}\right\}$ having definite values of the block spins $S_{1}$ and $S_{2}$. The following system of the nonlinear RG relations:

$$
\begin{gathered}
H=\frac{1}{4}\left[\Psi^{(++)}-\Psi^{(--)}\right], \\
K=\frac{1}{24}\left[\Psi^{(++)}+\Psi^{(--)}-2 \Psi^{(+-)}\right], \\
g=\frac{1}{8}\left[\Psi^{(++)}+\Psi^{(--)}+2 \Psi^{(+-)}\right],
\end{gathered}
$$

with spin functions $\Psi^{( \pm \pm)} \equiv \Psi\left(S_{1}= \pm 1, S_{2}= \pm 1\right)$ defines completely the RG transformation. The functions have the following symmetry properties:

$$
\begin{gathered}
\Psi^{(+-)}(h, k)=\Psi^{(-+)}(h, k), \\
\Psi^{(--)}(h, k)=\Psi^{(++)}(-h, k) .
\end{gathered}
$$

The most important property of the RSRG transformation is the existence of fixed points. The fixed points are determined by the conditions: $H=h, K=k$. From the symmetry of the first equation of Eqs. (17) it is obvious that all fixed points are located on the $k$-axis $\left(h_{c}=0\right)$. The nontrivial (i.e., $\left.k_{c} \neq 0\right)$ unstable fixed points of Eqs. (17) correspond to the critical points of the Hamiltonian, Eq. (11). To determine stability of a fixed point one must investigate the properties of the RSRG transformation at this point. In the close vicinity of the fixed point the RSRG transformation is reduced to the corresponding linear form

$$
\left(\begin{array}{c}
H \\
K-k_{c}
\end{array}\right)=\left(\begin{array}{ll}
\frac{\partial H}{\partial h} & \frac{\partial H}{\partial k} \\
\frac{\partial K}{\partial h} & \frac{\partial K}{\partial k}
\end{array}\right)\left(\begin{array}{c}
h \\
k-k_{c}
\end{array}\right) .
$$

Here all derivatives are computed at the critical point. The matrix of derivatives describes the linear response of the renormalized parameters, $(H, K)$ on variations of the original values $(h, k)$ around the fixed point $\left(0, k_{c}\right)$. This matrix has two eigenvalues: $\lambda_{1}$ and $\lambda_{2}$. The behavior of the thermodynamic quantities in the critical point is controlled by the eigenvalues, which are related to the RSRG critical exponents $y_{i}=d \ln \lambda_{i} / \ln L$. Only the relevant $\left(y_{i}>0\right)$ eigenvalues lead to the nonanalytical singular behavior of the thermodynamic quantities. In this case the RSRG transformation takes away the system from the fixed point, which means that the point is unstable. The RSRG transformations have one, socalled temperaturelike, eigenvalue (with exponent $y_{t}$ ), and one, fieldlike eigenvalue (with exponent $y_{h}$ ). The critical exponents for the Ising spin model are also known ${ }^{17}$

$$
y_{t} \approx 1.5868, \quad y_{h} \approx 2.4816 \text {. }
$$

As was shown by Nauenberg and Nienhuis ${ }^{19,20}$ the free energy of the system $F(h, k)$ for any values of its arguments can be evaluated in the series of sequential RSRG transformations of the original Hamiltonian

$$
F(h, k)=\sum_{m=1}^{\infty} L^{-m} g_{m} .
$$

Here $H_{m}\left(H_{m-1}, K_{m-1}\right), K_{m}\left(H_{m-1}, K_{m-1}\right)$, and $g_{m}\left(H_{m-1}, K_{m-1}\right)$ are the parameters of the $m$ th RSRG transformation; $H_{0}=h$, $K_{0}=k$.

In this work we have investigated finite lattices consisting of two block spins with periodic boundary conditions (every lattice consists of $2 L$ different site spins or 2 block spins). We considered more than 70 RSRG transformations with different blocks. There are some families of the transformations: $1 D$ blocks-lines of site spins, $2 D$ rectangles and $3 D$ "bricks." The linear block transformations are denoted as 1 $\times 1 \times n$ with $n=2, \ldots, 32$. The RSRG transformations with $2 D$ blocks $1 \times m \times n$ have $m=2, \ldots, 4$ and $n=2, \ldots, 16(m n$ $\leqslant 30$ ). The three-dimensional blocks have dimensions $2 \times 2$ $\times n$ with $n=2, \ldots, 6$, cubes $3 \times 3 \times 3$ and some "diagonal" blocks, like block $5 \times 2$ shown in Fig. 1 .

All RSRG transformations have some general properties. All transformations besides the smallest ones have F fixed points $\left(k_{c}>0\right)$ and many of them also have fixed points in the $\mathrm{AF}$ region $\left(k_{c}<0\right)$. The critical values of the interaction parameter approach the exact value as $L$ is increased. The best results are obtained for the three-dimensional blocks: All $2 \times 2 \times n, 3 \times 3 \times 3$ and diagonal blocks yield fairly good properties in the $\mathrm{F}$ region. In general, the critical properties of the transformations in the $\mathrm{F}$ region are superior to those in the $\mathrm{AF}$ region. The description of $\mathrm{AF}$ phase transitions is poor. There is no RSRG transformation capable of its quantitative description. 
For the cubic lattice the number of spins grows fast for the symmetrical cubic blocks, which are the best candidates for the RSRG transformation capable to describe AF phase transitions. Cubes with even $L=8,64, \ldots$ are unsuitable for AF interaction in principle and $L=125$ was considered too big. The critical properties of the RSRG transformations do not behave in a regular way. The location of the fixed points and values of the critical parameters are not monotonic functions of the block size $L$. As mentioned, for blocks with even $L$ one must determine the block spin for the spins configurations with zero sum of the site spins. This condition gives some freedom in distribution of these configurations. The properties of the RSRG transformations are sensitive to the distribution also.

Due to the symmetry properties of Eq. (19) any RSRG transformation is determined by two spin functions $\Psi^{(++)}$and $\Psi^{(+-)}$only. In general, they can be written in the following form:

$$
\Psi^{( \pm \pm)}(h, k)=\ln \left\{\sum_{i=1}^{n^{( \pm \pm)}} A_{i}^{( \pm \pm)} \exp \left[B_{i}^{( \pm \pm)} h+C_{i}^{( \pm \pm)} k\right]\right\}
$$

where all coefficients are integers ranging in the well-defined limits

$$
\begin{gathered}
B_{i}^{(++)}=0,2, \ldots, 2 L \\
B_{i}^{(--)}=-L,-L+2, \ldots, L-2, L \\
C_{i}^{( \pm \pm)}=-6 L,-6 L+4, \ldots, 6 L-4,6 L \\
\sum_{i=1}^{n^{( \pm \pm)}} A_{i}^{( \pm \pm)}=2^{2 L-2}
\end{gathered}
$$

Once determined, the two three-column tables (matrices) $\left\{A_{i}^{ \pm \pm}, B_{i}^{ \pm \pm}, C_{i}^{ \pm \pm}\right\}$can be used for the evaluation of the free energy and its derivatives for any values of the magnetic field and particle interaction (temperature). For big blocks with $L>20$ the corresponding summations over all configurations of spins entering the cluster of two blocks in order to determine the coefficients in these tables may be computationally expensive. The approach proposed by $\operatorname{Binder}^{23}$ (see also Refs. 24 and 25) offers a solution. The only modification required is to check the sum of spin values in every block and corresponding distribution of the spin configurations between the tables.

To evaluate the quality of the transformations we have determined their critical parameters. They include critical values of the spin-spin pair interaction parameter, $k_{c}$, critical exponents $y_{h}$ and $y_{t}$, critical entropy $S_{c}$, and internal energy $U_{c}$. Reference values of these quantities were obtained by high-temperature series ${ }^{26}$

$$
S_{c} / k_{B} \approx 0.558, \quad-U_{c} / k_{B} T_{c} \approx 0.220 .
$$

The critical values for the best RSRG transformations are collected in Table I.

The relative error of the most accurate RSRG transformations with three-dimensional blocks for attractive particle interaction remains within several percent. It seems quite natu- ral that the closer these parameters are to the exact values the better is the transformation. This criterion is well-suited for the strongly divergent thermodynamic quantities like specific heat and density fluctuations in the vicinities of the critical points. Outside of these regions many transformations give close results and the best ones yield almost the same data.

\section{THE MONTE CARLO SIMULATIONS}

The Monte Carlo technique is a powerful and reliable method which is used widely to study the complex phenomena especially where analytical approaches are unavailable or work badly. Due to its simplicity and the availability of powerful supercomputers the MC simulations have been widely used for investigations of kinetic properties of lattice gas models. We applied the MC approach for calculations of the tracer and jump diffusion coefficients and some thermodynamic quantities of the particle system.

The system represented by the Hamiltonian Eq. (11) is realized by a simple cubic array of $M \times M \times M$ sites with periodic boundary conditions. We assume that all sites are separated by wells of the periodic potential. The saddle point energies (describing the barriers which need to be overcome by diffusion particles) are uniformly given by a common value, $\varepsilon$, throughout the whole lattice unaffected by particleparticle interactions. Initial lattice gas configurations are generated by distributing $\theta M^{3}$ particles at random on the lattice.

The MC algorithm corresponds to Metropolis importance sampling (standard MC): Pick at random an initial site $i$ out of all eligible (filled) sites. Select an adjacent final site $f$ randomly. If the destination is vacant, a jump can occur with a probability

$$
P_{i f}=\nu_{i f} / \nu_{\max }
$$

where $\nu_{i f}$ is the jump frequency, given by Eq. (7) and $\nu_{\max }$ is its maximum value

$$
\nu_{\max }= \begin{cases}\nu \exp (-\varepsilon), & \text { if } \varphi<0, \\ \nu \exp (-\varepsilon+5 \varphi), & \text { if } \varphi>0 .\end{cases}
$$

For high-density cases $(\theta>0.5)$ the roles of initial and final sites can be reversed. One Monte Carlo step (MCS) corresponds to $\min (1-\theta, \theta) M^{3}$ interrogations of the eligible lattice sites in random order. The dimension of the cubic lattice was $M=32$. In order to ensure the collection of statistics over uncorrelated configurations sampling a configuration was followed by $400 \mathrm{MC}$ steps. However, it turned out that the considered quantities hardly depended on the number of $\mathrm{MC}$ steps (ranging from 200 to $1000 \mathrm{MC}$ steps separating the configuration sampling). A total number of 40000 configurations was collected.

Although the bookkeeping overhead is small, this MC algorithm suffers from the small transition probability $P_{\text {if }}$ at low temperatures where the fraction of rejected jumps is high. The inefficiency for low particle jump transition probabilities can be overcome using the $N$-fold way $\mathrm{MC}$ algorithm. ${ }^{27}$ In contrast to standard MC where we consider fixed time intervals at which a jump may occur with a certain probability, the $N$-fold way MC selects randomly a particle 
TABLE I. Compilation of the ferromagnetic critical values for the different RSRG blocks studied in the present work. The first column shows dimensions of blocks, the second is the number of site spins in the block. The third and forth-number of terms in the spin functions $\Psi^{++}$and $\Psi^{+-} ; k_{c}$ is the value of the spin pair interaction parameter in the F critical point; $y_{h}$ and $y_{t}$ are the corresponding critical exponents; $\epsilon$ is the relative error of the critical value of the pair interaction parameter (in \%); $S_{c}$ and $U_{c}$ are the values of entropy and internal energy in the critical point.

\begin{tabular}{|c|c|c|c|c|c|c|c|c|c|}
\hline $\begin{array}{l}l \times m \times n \\
\text { exact }\end{array}$ & $L$ & $n^{++}$ & $n^{+-}$ & $\begin{array}{c}k_{c} \\
0.22165\end{array}$ & $\epsilon(\%)$ & $\begin{array}{c}y_{h} \\
2.4816\end{array}$ & $\begin{array}{c}y_{t} \\
1.5868\end{array}$ & $\begin{array}{l}S_{c} / k_{B} \\
0.588\end{array}$ & $\begin{array}{c}-U_{c} / k_{B} T_{c} \\
0.220\end{array}$ \\
\hline $1 \times 2 \times 7$ & 14 & 196 & 232 & 0.2237 & 0.91 & 2.15 & 0.41 & 0.510 & 0.304 \\
\hline $1 \times 2 \times 6$ & 12 & 136 & 153 & 0.2067 & 6.75 & 2.07 & 0.40 & 0.549 & 0.241 \\
\hline $1 \times 3 \times 4$ & 12 & 128 & 177 & 0.2038 & 8.04 & 2.18 & 0.61 & 0.571 & 0.203 \\
\hline $1 \times 2 \times 8$ & 16 & 266 & 305 & 0.2371 & 6.97 & 2.20 & 0.44 & 0.478 & 0.359 \\
\hline $1 \times 4 \times 4$ & 16 & 234 & 314 & 0.2136 & 3.64 & 2.25 & 0.69 & 0.555 & 0.231 \\
\hline $1 \times 1 \times 18$ & 18 & 408 & 474 & 0.2337 & 5.44 & 1.69 & 0.12 & 0.490 & 0.428 \\
\hline $1 \times 3 \times 6$ & 18 & 345 & 467 & 0.2245 & 1.31 & 2.28 & 0.65 & 0.523 & 0.281 \\
\hline $1 \times 4 \times 5$ & 20 & 406 & 567 & 0.2215 & 0.08 & 2.29 & 0.74 & 0.539 & 0.258 \\
\hline $2 \times 2 \times 2$ & 8 & 33 & 54 & 0.2062 & 6.95 & 2.37 & 0.93 & 0.555 & 0.205 \\
\hline $2 \times 2 \times 3$ & 12 & 103 & 165 & 0.2058 & 7.16 & 2.34 & 0.88 & 0.564 & 0.198 \\
\hline $2 \times 2 \times 4$ & 16 & 223 & 332 & 0.2108 & 4.89 & 2.36 & 0.89 & 0.554 & 0.214 \\
\hline $2 \times 2 \times 5$ & 20 & 391 & 583 & 0.2164 & 2.36 & 2.38 & 0.89 & 0.539 & 0.237 \\
\hline $2 \times 2 \times 6$ & 24 & 608 & 870 & 0.2217 & 0.03 & 2.40 & 0.89 & 0.522 & 0.262 \\
\hline $2 \times 2 \times 3^{a}$ & 15 & 181 & 295 & 0.2296 & 3.56 & 2.57 & 1.23 & 0.493 & 0.299 \\
\hline $3 \times 2 \times 5^{a}$ & 22 & 440 & 652 & 0.2104 & 5.08 & 2.34 & 0.88 & 0.560 & 0.207 \\
\hline $3 \times 3 \times 3$ & 27 & 654 & 1060 & 0.2263 & 2.11 & 2.56 & 1.29 & 0.512 & 0.273 \\
\hline
\end{tabular}

a"Diagonal" square block as shown upper left in Fig. 1.

jump out of all possible events and subsequently associates the number of clock ticks that must have passed for the event to take place. So the transition probability does not result in rejected particle jumps but to a different amount of elapsed time, making the algorithm particularly efficient at low temperatures. However, it is necessary to classify all possible events into groups of equal transition probability. In this case there are only six different classes, depending upon the number of occupied sites surrounding the initial site. By drawing a random number, an event class is selected, another random number picks a specific event from the selected class and finally drawing a third random number produces the number of clock ticks that have passed. Details are given in Refs. 28 and 29. The bookkeeping overhead for updating the class association of events, however, is substantial. To suppress the correlation between subsequently sampled configurations, configurations were sampled at certain time intervals. Again 40000 configurations were collected and the dependence of the results upon time interval selection and in comparison to those of the standard MC was investigated.

The MC codes have been parallelized using MPI by dividing the entire workload of density dependent configuration sampling into independent tasks, so that the overall communication load is negligible.

The absorption isotherms, $\mu(\theta)$, have been calculated using the method of local states. ${ }^{30}$ The energy of any absorbed particle can take the following seven values: $E_{i}=n \varphi$, $n=0,1, \ldots, 6$, depending on the number of $n n$ s. The energies define the local states of the particles. The set of conjugate states is determined for holes (empty sites). All conjugate states have zero energy. The frequencies of occurrence (ensemble average populations) of the $i$ th local state and its conjugation are denoted as $\sigma_{i}$ and $\sigma_{i}^{\prime}$, respectively. The frequencies are related by the condition of the detailed balance as follows:

$$
\mu=\ln \left(\sigma_{i} / \sigma_{i}^{\prime}\right)+E_{i}, \quad i=1, \ldots, 6
$$

The isothermal susceptibility $\chi_{T}$ is the mean-square particle density fluctuations. They are measured in a small probe region which consists of $N_{p}$ sites. The shape of the probe region may be arbitrary: A smaller cube or a slice of the lattice $M \times M \times n$ with $n=1,2,4, \ldots$. For isotropic lattices it does not matter. The average over the initial configurations squared fluctuations of the number of particles in this region $(\delta N)^{2}$ is related to the isothermal susceptibility

$$
\chi_{T}=\frac{N(\delta N)^{2}}{N_{p}\left(N-N_{p}\right)}
$$

The chemical diffusion coefficient, $D_{c}$, which is a many particle diffusion coefficient, has been determined via the KuboGreen equation, Eq. (9), relating the jump and chemical diffusion coefficients.

\section{RESULTS AND DISCUSSION}

Using the most accurate RSRG transformations we have calculated the density dependencies of the thermodynamics quantities of interest for repulsively and attractively interacting particles and compared them with the MC simulation results. 


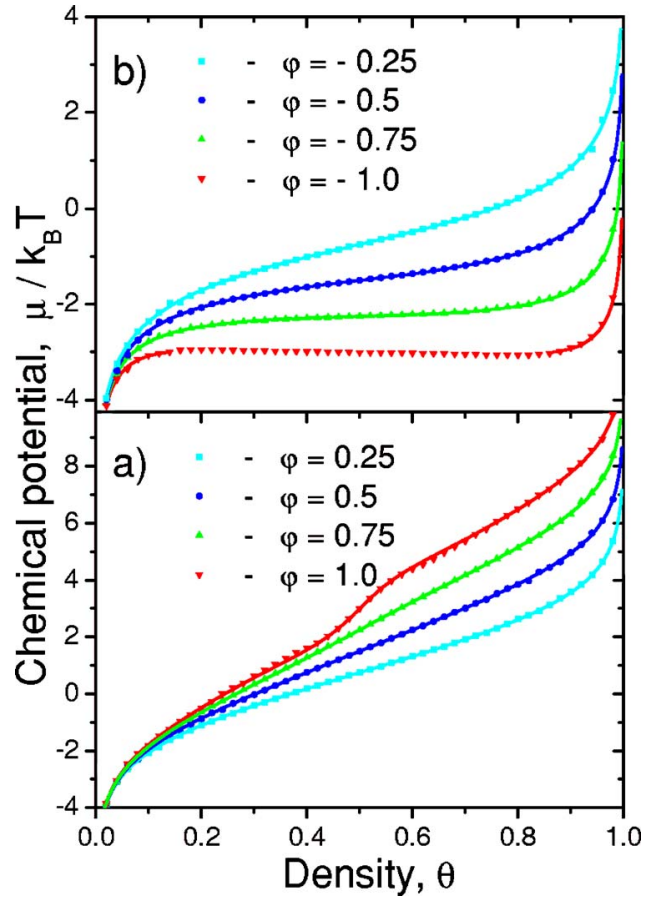

FIG. 2. (Color online) Absorption isotherms ( $\mu$, vs $\theta$ ) for some representative values of the interaction parameter $\varphi$ as indicated: (a) repulsive interaction, (b) attractive interaction. Solid lines and symbols denote the RSRG and MC data, respectively.

At first we consider thermodynamic quantities related to the first derivatives of the free energy over its arguments. Using the most accurate RSRG transformation (cluster of two cubes $3 \times 3 \times 3)$ we have calculated absorption isotherms, $\mu(\theta)$, for repulsion and attraction between the particles (cf. Fig. 2). At high temperatures the dependencies are close to the Langmuir case $(\varphi=0)$

$$
\mu / k_{B} T=\ln [\theta /(1-\theta)] .
$$

As the temperature decreases, the behavior of the dependencies becomes quite different. For repulsive interaction a peculiarity appears at $\theta=0.5$ which indicates the formation of an ordered structure. The isotherm has a very steep slope at this density. The chemical potential varies in a wide region but the particle density remains almost constant. For attractive interaction a horizontal plateau appears at the critical temperature which turns to a jump of the density. The jump corresponds to the first-order phase transition between two lattice gas phases having different densities. The agreement between RSRG and MC data is excellent for all temperatures and densities studied.

Another thermodynamic quantity related to the first derivative of the free energy over the interaction parameter is the correlation function $P_{00}$ which is needed to calculate the jump and chemical diffusion coefficients. The density dependencies $P_{00}(\theta)$ are smooth curves (see Fig. 3). At high temperatures they are close to the mean-field result $P_{00}=(1$ $-\theta)^{2}$. Again, RSRG and MC data agree well over the whole range of temperatures and coverage.

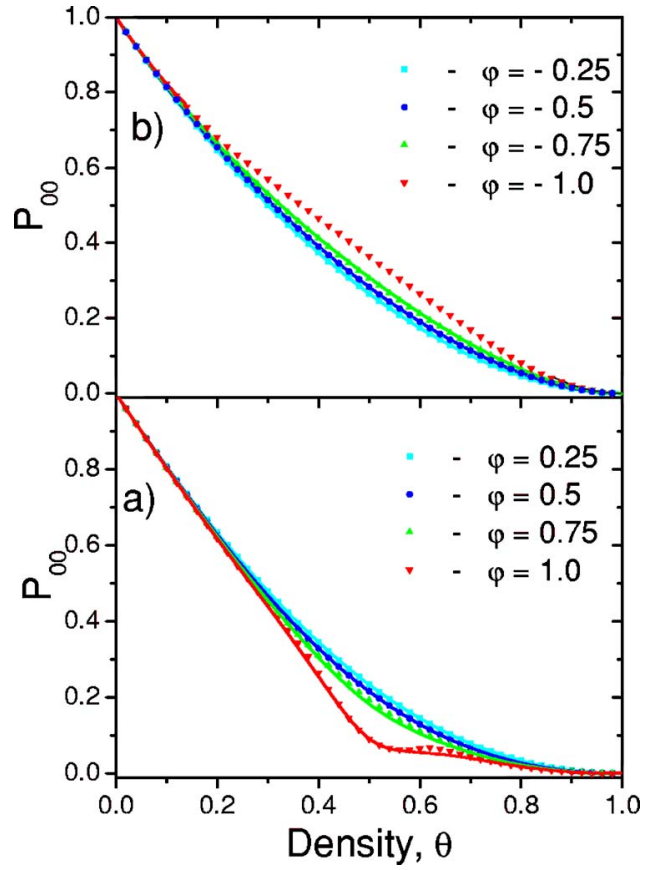

FIG. 3. (Color online) The density dependencies of the pair correlation function, $P_{00}$. Solid lines and symbols denote the RSRG and MC data.

The quantity being the most sensitive to phase transitions is the second derivative of the free energy over the chemical potential or the isothermal susceptibility

$$
\chi_{T}=\left(\frac{\partial^{2} F}{\partial h^{2}}\right)_{T}=N^{-1} \sum_{i, j}\left(n_{i}-\theta\right)\left(n_{j}-\theta\right) .
$$

The density dependencies of the isothermal susceptibility are plotted in Fig. 4. For high temperatures the mean square of density fluctuations equals $\theta(1-\theta)$. If the temperature is lowered the behavior of susceptibility depends on the sign of the interaction parameter $\varphi$. The lateral repulsion decreases the density fluctuations keeping particles apart from each other. The density fluctuations are strongly suppressed at $\theta=0.5$ [see Fig. 4(a)]. In the ordered phase any density disturbance (i.e., displacement of a particle from its "right" position in the filled sublattice to any site of the empty sublattice) significantly increases the free energy of the system and is thermodynamically unfavorable. As the density does not equal 0.5 , there are fluctuations of the nonstoichiometric nature that do not require additional energy for their existence and cannot be removed by the particle jumps. Therefore, $\chi_{T}$ increases when $\theta$ deviates from the stoichiometric value and the dependencies $\chi_{T}(\theta)$ have deep and narrow minimum at low temperatures but remains analytical. There is a rather good agreement between RSRG and MC data for high temperatures but noticeable deviations appear at low temperatures.

Attraction causes the overall growing of the density fluctuations, keeping particles together and inhibiting the relaxation of the density disturbances. The dependencies exhibit a sharp maximum at half coverage, growing to infinity as $\varphi$ $\rightarrow \varphi_{c} \approx 0.88662$. Any density disturbance relaxes slower and 


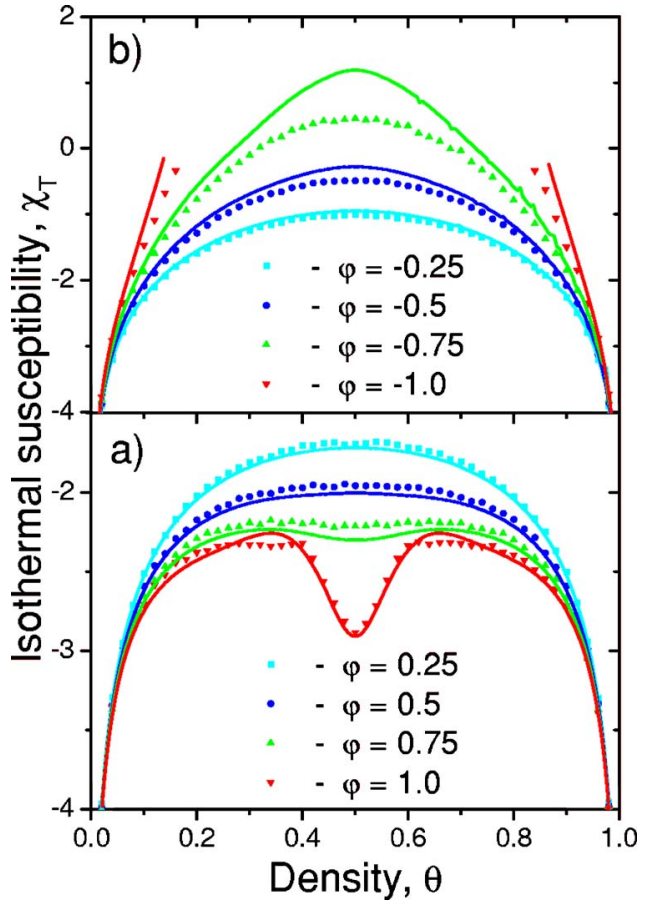

FIG. 4. (Color online) Mean square density fluctuations, $\ln \chi_{T}$, vs $\theta$. Solid lines and symbols denote the RSRG and MC data, respectively.

slower as the interaction tends to its critical value. The system becomes unstable in the critical point and fluctuations are strongly divergent. The particle distribution over lattice is no more homogeneous: Drops of dense liquid phase arise in the lattice gas phase. For $|\varphi|>\varphi_{c}$ a broad density region $\theta_{c}<\theta<1-\theta_{c}$ is inaccessible for the RSRG approach. One cannot fix the density $\theta$ inside the interval of the inhomogeneity $\left(\theta_{c}, 1-\theta_{c}\right)$, and calculate the thermodynamic quantities and diffusion coefficients, $D_{j}, D_{c}$, in the mixture of phases with different densities. It is the region where any stable thermodynamic state is really impossible.

The coincidence between the RSRG and MC data in Fig. 4(b) is good for high temperatures but again there are visible and regular deviations between the RSRG and MC data which grow as the temperature is lowered. It should be noted that irrespective of the method the isothermal susceptibility is the most challenging property to compute. Due to its singular behavior the data are more sensitive to the choice of the RSRG blocks. The (infinite) growth of the density fluctuations is controlled by the critical exponents, so the nearness of their values to the exact ones becomes important. There is a well-known scaling dependence describing the critical divergence of the isothermal susceptibility

$$
\chi_{T} \sim\left(T-T_{c}\right)^{-\gamma} \quad \text { as } \quad T-T_{c} \rightarrow+0,
$$

where $\gamma=\left(2 y_{h}-d\right) / y_{t}>0, d=3$ and $\theta=0.5$.

One can determine easily the critical exponent $\gamma$ using the RSRG values for $y_{t}$ and $y_{h}$ listed in Table I.

$\mathrm{MC}$, on the other hand, requires longer samplings and needs to average over more initial configurations in order to obtain smooth isothermal susceptibility.

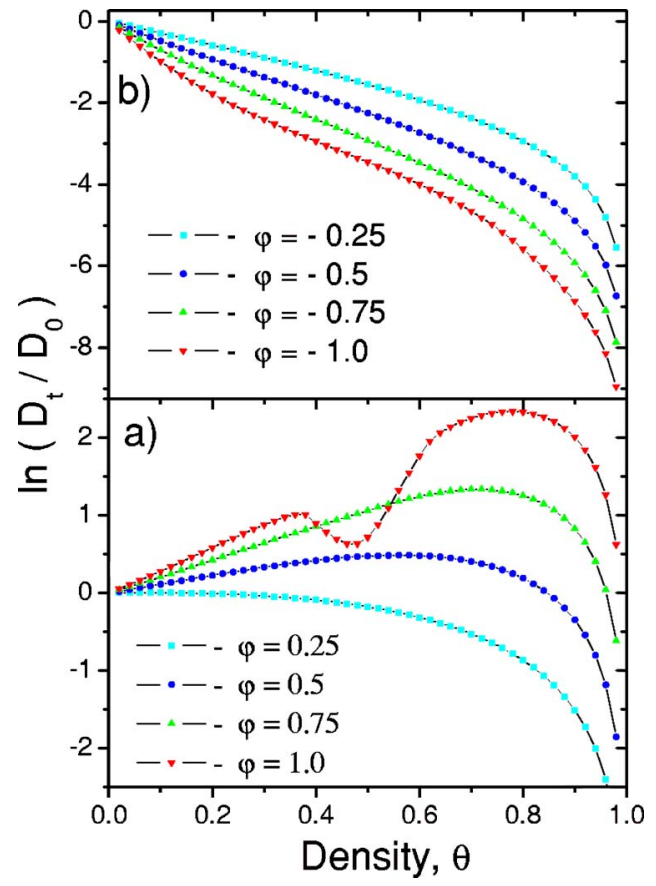

FIG. 5. (Color online) The density dependencies for the tracer diffusion coefficient, $D_{t}$. The MC data only.

In Fig. 5 we show the density dependencies of the tracer diffusion coefficient, $D_{t}$, for repulsive and attractive interactions, in Figs. 5(a) and 5(b), respectively. There are the MC data only. As mentioned above, the tracer diffusion coefficient describes the behavior of a single tagged particle. At high temperatures $D_{t}(\theta)$ is a monotonic decreasing nonlinear function of the particle density, $\theta$. If the temperature is lowered, the behavior becomes nonmonotonic due to the increasing role of the lateral particle interaction. As usual, the repulsive interaction increases particle migration and attraction inhibits its mobility. Strong repulsion causes phase transition from the disordered state to the ordered phase, when particles occupy lattice sites in alternating order. Then every occupied site is surrounded by its six empty $n n$ s. Diffusion in this phase is inhibited and the dependence $D_{t}(\theta)$ dipped in a wide region of densities where the particle system is in the ordered state. Attractive interaction does not change qualitatively the density dependence of the tracer diffusion coefficient. The tracer diffusion coefficient, $D_{t}$, falls to zero as $\theta \rightarrow 1$. The diffusion of tracers is impossible in the completely occupied lattice.

The density dependencies of the jump diffusion coefficient $D_{j}$ are plotted in Fig. 6 for the same values of the interaction parameter. The plots are qualitatively similar to those of the tracer diffusion coefficient. The solid lines and symbols represent the RSRG and MC data, respectively, agreeing almost perfectly. Even in the immediate vicinity of the critical temperature $(\varphi=0.75$ and $\varphi=1)$ the numerical data and analytical curves are close. For the ordered phase $(0.4<\theta<0.6$ at $\varphi=1)$, however, they noticeably deviate. In this region the particles are ordered and particle jumps became strongly correlated.

The RSRG dependencies of the chemical diffusion coefficient on the density with the MC data are plotted in Fig. 7. 


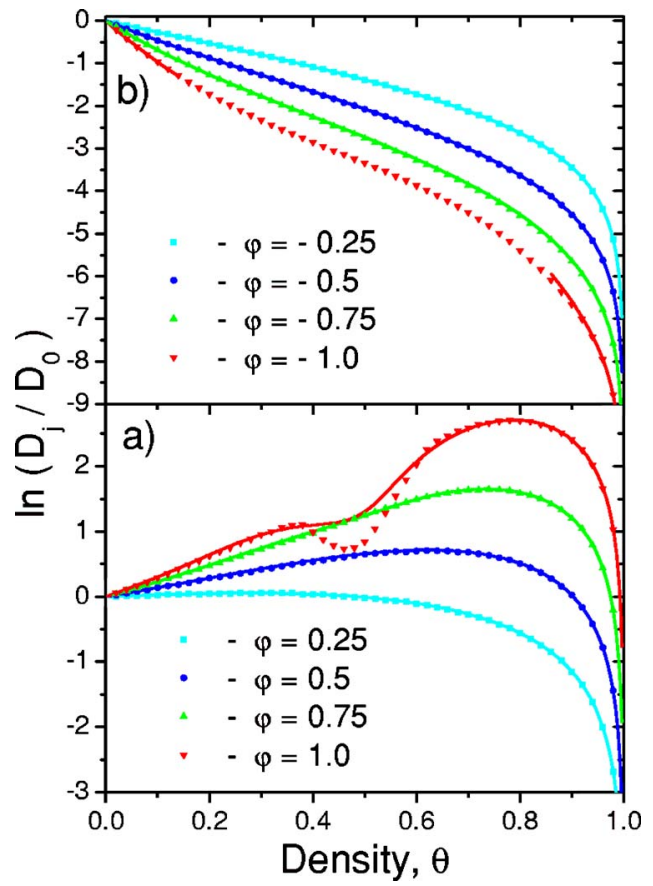

FIG. 6. (Color online) Jump diffusion coefficient, $D_{j}$, vs $\theta$. Solid lines and symbols denote the RSRG and MC data, respectively.

In the limits of $\theta \rightarrow 0,1$, a jumping particle has either zero or five $n n \mathrm{~s}$, respectively. Therefore, the limiting values of $D_{c}$ are the following:

$$
\begin{gathered}
\lim _{\theta \rightarrow 0} D_{c}=D_{0}, \\
\lim _{\theta \rightarrow 1} D_{c}=D_{0} \exp (5 \varphi) .
\end{gathered}
$$

The density dependencies for repulsive interaction are shown in Fig. 7(a). In the disordered phase the $\ln D_{c} / D_{0}$ grows almost linearly with the density as the mean number of $n n$ s for any jumping particle is also growing. It is interesting to note that qualitatively in the same behavior one can see at densities slightly less then 1 . In this region the relaxation of the fluctuations proceeds by the diffusion of holes. Due to the evident Hamiltonian's symmetry between particles and holes, the repulsion energy for holes is also equal to $\varphi$. Therefore, the diffusion coefficient grows with density of holes $\theta_{h}=1-\theta$. In the ordered phase the diffusion is inhibited and dependencies have a wide minimum. The RSRG data coincide rather well with the numerical results in disordered lattice gas but deviate markedly in the ordered phase as in the case of the jump diffusion coefficient due to the correlations between the particle jumps in the ordered phase.

For attractive interaction the behavior of the chemical diffusion coefficient in the gas phase $\left(\theta<\theta_{c}\right.$ and $\left.\theta>1-\theta_{c}\right)$ is qualitatively the same as in the previous case: $\ln D_{c} / D_{0}$ decreases almost linearly with the coverage $\theta$, as the mean number of $n n \mathrm{~s}$ is increasing (for $\theta<\theta_{c}$ ) and increases almost linearly as the hole density is decreased (for $\theta \rightarrow 1$ ). When approaching the critical line separating the homogeneous and inhomogeneous phases the fluctuations grow up to infinity

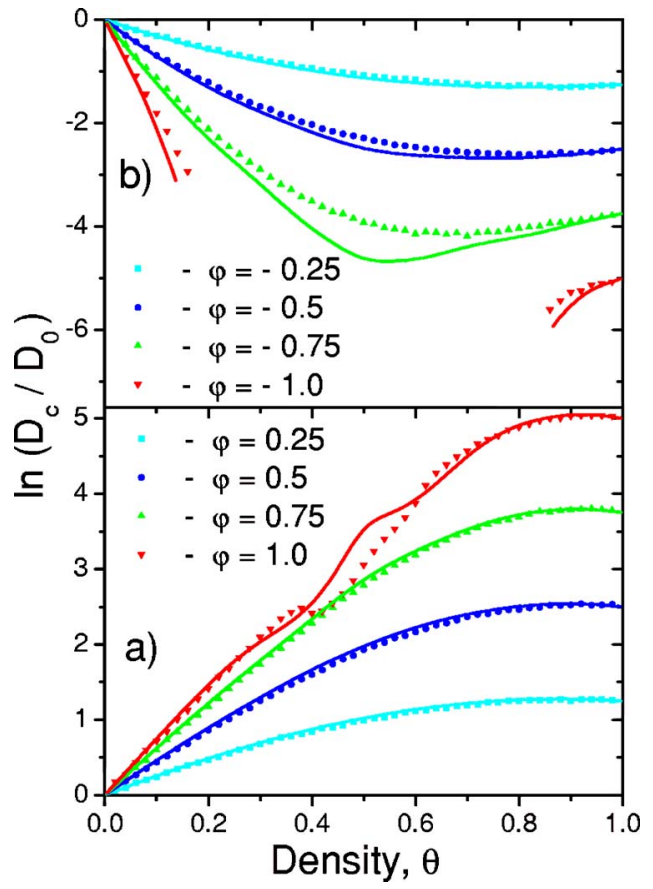

FIG. 7. (Color online) Chemical diffusion coefficient, $D_{c}$, vs $\theta$. Solid lines and symbols denote the RSRG and MC data, respectively.

and cause the critical slowdown of the chemical diffusion coefficient to zero. Diffusion relaxation of the density disturbances is switched off and the system becomes inhomogeneous. The coincidence between the RSRG and MC data is good for small densities and when $\theta$ approaches 1 . The deviations appear as the temperature is lowered for $\theta \sim 0.5$. There are deviations caused by poor quality of the RSRG data for the isothermal susceptibility as we use them in calculations of $D_{c}$. It should be noted that neither the chemical diffusion coefficient in the MC simulations nor the jump diffusion coefficient in the analytical approach are calculated in the direct way. Then the Kubo-Green relation [Eq. (9)] is used to obtain data for these coefficients. But the coincidence between the values for $D_{j}$ directly measured by the MC simulations and those calculated via Eqs. (8) and (9) is almost perfect for all temperatures and densities. It means that Eqs. (8) and (9) are valid even in the vicinity of the critical point of the first-order phase transition.

Summing up we should say that the interplay between the particle lateral interaction and their transport coefficients is rather complex. The lateral repulsion-attraction exponentially increases-inhibits the jump rates of the particles, thus drastically changing the behavior of the diffusion coefficients, whose values can vary by orders of magnitude at different particle densities. The more subtle memory effect, caused by an increased probability of the backward jumps, produces about $35 \%$ decrease of the tracer diffusion only. Nevertheless, the strong interaction can increase the correlation in the successive particle jumps. It occurs at temperatures below the critical point at the stoichiometric densities when the system of particles is almost perfectly ordered. The effects of interaction-increased jump rate and high probability of the backward jump compensate each other to some degree and 
the diffusion coefficient decreases in this region. Strong attraction changes the particle migration completely. The density disturbances do not relax in the ordinary way, so that this description does not apply to such thermodynamically unstable situations.

\section{SUMMARY}

We have investigated a number of RSRG transformations on the simple cubic lattice with blocks of different size and symmetries. In general the accuracy of the method increases with the number of sites in the RSRG block. The most accurate results have been obtained for the large blocks $3 \times 3$ $\times 3$ and diagonal blocks containing 15 sites each for the pairwise attractive interaction. The critical temperature, critical magnetic field, and critical indices coincide well with the known values for these parameters. The RSRG transformations are rather well-suited to describe the critical properties of the ferromagnetic spin model, but fail to accurately reproduce the AF critical behavior.

Using the RSRG method we have calculated density dependencies of the isothermal susceptibility and jump and chemical diffusion coefficients. All these quantities have been compared to MC simulation results. The results ob- tained by these entirely different methods, agree well over the entire density region at different temperatures except for the vicinity of the critical points.

The RSRG approach does not require advanced mathematics and is computationally inexpensive and can be carried out on an ordinary PC straight from the shelf. Its disadvantage is the absence of the regular behavior of the RSRG transformations which adds an element of trial and error.

Summing up all results we can conclude that the RSRG method can be used successfully for investigations of the thermodynamic properties of the lattice gas and spin systems. The thermodynamic quantities and in some cases even kinetic coefficients can be calculated accurately for lattice gas systems with strong lateral interaction.

\section{ACKNOWLEDGMENTS}

This work has been supported by the Institutional Research Plan AV CR No. AV0Z 10100522 and project 1QS100100563 AV CR. We gratefully acknowledge computer time on the IBM p690 supercomputer cluster at the John-von-Neumann Institute for Computing at the Research Centre Jülich (NIC Project No. k2720000) where the Monte Carlo simulations have been carried out.
*Corresponding author. FAX: 420286581448. Electronic address: taras@fzu.cz

${ }^{1}$ R. Kutner, K. Binder, and K. W. Kehr, Phys. Rev. B 26, 2967 (1982).

${ }^{2}$ A. Danani, R. Ferrando, E. Scalas, and M. Torri, Int. J. Mod. Phys. B 11, 2217 (1997).

${ }^{3}$ A. Danani, R. Ferrando, E. Scalas, and M. Torri, Surf. Sci. 402404, 281 (1998).

${ }^{4}$ A. Danani, R. Ferrando, E. Scalas, and M. Torri, Surf. Sci. 409, 117 (1998).

${ }^{5}$ A. A. Tarasenko, L. Jastrabik, and C. Uebing, Phys. Rev. B 57, 10166 (1998).

${ }^{6}$ A. A. Tarasenko, L. Jastrabik, F. Nieto, and C. Uebing, Phys. Rev. B 59, 8252 (1999).

${ }^{7}$ A. A. Tarasenko, F. Nieto, L. Jastrabik, and C. Uebing, Phys. Rev. B 64, 075413 (2001).

${ }^{8}$ F. Nieto, A. A. Tarasenko, and C. Uebing, Europhys. Lett. 43, 558 (1998)

${ }^{9}$ A. A. Tarasenko, F. Nieto, and C. Uebing, Phys. Chem. Chem. Phys. 1, 1583 (1999).

${ }^{10}$ A. A. Chumak and A. A. Tarasenko, Surf. Sci. 91, 694 (1980).

${ }^{11}$ D. A. Reed, and G. Ehrlich, Surf. Sci. 102, 558 (1981); Surf. Sci. 105, 603 (1981).

${ }^{12}$ V. P. Zhdanov, Elementary Physicochemical Processes on Solid Surfaces (Plenum, New York, 1991).

${ }^{13}$ P. Argyrakis, Y. G. Groda, G. S. Bokun, and V. S. Vikhrenko, Phys. Rev. E 64, 066108-11 (2001).

${ }^{14}$ P. Argyrakis and A. A. Chumak, Phys. Rev. B 66, 054303 (2002).
${ }^{15}$ Y. G. Groda, P. Argirakis, G. S. Bokun, and V. S. Vikhrenko, Eur. Phys. J. B 32, 327 (2003).

${ }^{16}$ T. Ala-Nissila, R. Ferrando, and S. C. Ying, Adv. Phys. 51, 949 (2002).

${ }^{17}$ Y. Deng and H. W. J. Blöte, Phys. Rev. E 68, 036125 (2003).

${ }^{18}$ Th. Niemeyer and J. M. J. van Leeuwen, Physica (Amsterdam) 71, 17 (1974).

${ }^{19}$ M. Nauenberg and B. Nienhuis, Phys. Rev. Lett. 33, 1598 (1974).

${ }^{20}$ B. Nienhuis and M. Nauenberg, Phys. Rev. Lett. 35, 477 (1975).

${ }^{21}$ Th. Niemeyer and J. M. J. van Leeuwen, in Phase Transitions and Critical Phenomena, edited by C. Domb and M. S. Green (Academic, New York 1976), Vol. VI, Chap. 7.

${ }^{22}$ B. Nienhuis and M. Nauenberg, Phys. Rev. B 11, 4152 (1975).

${ }^{23}$ K. Binder, Physica (Amsterdam) 62, 508 (1972).

${ }^{24}$ G. Bhanot, J. Stat. Phys. 60, 55 (1990).

${ }^{25}$ G. Bhanot and S. Sastry, J. Stat. Phys. 60, 333 (1990).

${ }^{26}$ C. Domb, The Critical Point (Taylor and Fransis, London, 1996).

${ }^{27}$ A. B. Bortz, M. H. Kalos, and J. L. Lebovitz, J. Comput. Phys. 17, 10 (1975).

${ }^{28}$ F. M. Bulnes, V. D. Pereyra, and J. L. Riccardo, Phys. Rev. E 58, 86 (1998).

${ }^{29}$ M. A. Novotny, in Annual Reviews of Computational Physics IX, edited by D. Stauffer (World Scientific, Singapore, 2001), pp. 153-210 (also available at http://www.arxiv.org/cond-mat/ 0109182).

${ }^{30}$ Z. Alexandrowicz, J. Stat. Phys. 13, 231 (1975); J. Stat. Phys. 14, 1 (1976). 